\title{
ANÁLISE DAS CARACTERÍSTICAS CORPORAIS DO PEIXE Betta splendens
}

\section{ANALYSIS OF CORPORATE CHARACTERISTICS OF THE FISH Betta splendens}

\author{
Veruska Dilyanne Silva Gomes', Alda Lúcia de Lima Amâncio², Cacio Ribeiro \\ Cavalcanti $^{3}$, João Marcos Monteiro Batista ${ }^{4}$
}

1 - Doutorado em Zootecnia - Universidade Federal da Paraíba

2 - Professora Doutora da Universidade Federal da Paraíba - CCHSA/Centro de Ciências Humanas, Sociais e Agrárias /Departamento de Ciência Animal

3 - Zootecnista, doutorando em Engenharia Agrícola, Universidade Federal de Campina Grande

4 - Zootecnista, doutorando em Zootecnia, Universidade Federal do Goiás.

Autor para correspondência: veruska_sgomes@yahoo.com.br

\section{RESUMO:}

A piscicultura ornamental representa um mercado lucrativo. Uma característica marcante do setor é a variedade de espécies das quais, o Betta splendens se destaca como um dos peixes mais comercializados no cenário mundial. Mediante a importância da espécie e escassez de informações sobre o tema, a pesquisa foi desenvolvida com o objetivo de descrever características fenotípicas e a concentração de glicose sanguínea (dm/l) de acordo com o sexo, como também, identificar correlações entre peso corporal e medidas morfométricas do Betta splendens. Foram utilizados 80 peixes divididos em dois grupos, machos e fêmeas com 6 meses de vida. A coleta de dados consistiu na pesagem, medição (comprimento total e padrão, largura, altura e comprimento da cabeça) e coleta de sangue para análise da concentração de glicose. Os dados foram submetidos à análise de variância, as médias comparadas pelo teste t, e realizada análise de correlação de Pearson entre peso e características morfométricas. Os machos possuem maior comprimento total e de cabeça, enquanto as fêmeas apresentam maior largura. A glicose sanguínea permaneceu entre 50,40 e 51,11 dm/l para a espécie. Existe forte correlação positiva entre todas as variáveis analisadas, sendo a relação entre comprimento total e comprimento padrão a mais forte observada no grupo das fêmeas, e a relação entre comprimento total e comprimento de cabeça para machos. Conhecer o comportamento e relação entre características fenotípicas do Betta splendens pode contribuir para estudos visando seleção de características desejáveis nas espécies ornamentais, contribuindo com a formação de novas linhagens.

Palavras-chaves: aquariofilia, correlações, glicose sérica, piscicultura ornamental.

\begin{abstract}
:
Ornamental fish farming represents a lucrative market. A striking feature of the industry is the variety of species of which Betta splendens stands out as one of the most commercialized fish on the world stage. Through the importance of the species and the scarcity of information on the subject, the research was developed with the objective to describe phenotypic characteristics and blood glucose concentration ( $\mathrm{dm} / \mathrm{l})$ according to sex, as also to identify correlations between body weight and morphometric measurements of Betta splendens. It was used 80 fish divided in two groups, males and females with 6 months of life. Data collection consisted of weighing, measuring (total and standard length, width, height and head length) and blood collection for analysis of glucose concentration. Data were submitted to analysis of variance, the means were compared by the $t$ test at the
\end{abstract}


$5 \%$ probability level and, later, Pearson correlation analysis between weight and morphometric characteristics. Males have larger total length and head, while females have greater width. Blood glucose remained between 50,40 and 51,11 dm/l for the species. There is a strong positive correlation between all analyzed variables, with the ratio between total length and standard length being the strongest observed in the females group, and the relation between total length and head length for males. Knowing the behavior and relation between phenotypic characteristics of Betta splendens it can contribute to studies aiming at selecting desirable characteristics in ornamental species, contributing to the formation of new lineages.

KEYWORDS: aquarium fish, correlations, serum glucose, ornamental fish.

\section{INTRODUÇÃO}

A piscicultura ornamental vem crescendo consideravelmente devido, tanto à alta rentabilidade da comercialização de espécies nativas e exóticas destinadas ao abastecimento deste mercado, quanto ao aumento da demanda pelo consumidor final. Segundo Whittington e Chong (2007), o mercado do peixe ornamental obteve alto faturamento com a comercialização de cerca de um bilhão de peixes ornamentais. Neste setor, a variedade de espécies uma característica marcante do setor (MCDOWALL, 2004).

O peixe Betta splendens é uma das espécies ornamentais mais comercializadas no cenário mundial. Apresenta hábito alimentar carnívoro, sendo apontado por Cavalcanti et al. (2007) como uma alternativa biológica eficaz no combate ao mosquito transmissor da dengue, pela notável capacidade de predar as larvas presente na água.

A espécie é originária da Ásia, conhecida como "peixe lutador" devido ao seu comportamento territorialista e sua agressividade, principalmente observada entre os machos (FOSSE, et al., 2013). Morfologicamente, são caracterizados pela existência de diferenças fenotípicas relacionadas ao sexo, ou seja, dimorfismo sexual (FARIA et al., 2006).

O dimorfismo sexual começa a se tornar evidente a partir dos dois meses de idade, quando as nadadeiras dos machos se tornam mais desenvolvidas. Machos adultos de linhagens comerciais possuem nadadeiras expressivamente maiores do que as fêmeas, no entanto, algumas espécies nativas não possuem diferenças corporais facilmente detectáveis (FARIA et al., 2006).

O conhecimento sobre correlações entre características fenotípicas é de grande interesse para a realização de programas de melhoramento genético de uma espécie, permitindo avaliar o grau pelo qual duas caraterísticas tendem a mudar juntas (OLIVEIRA et al., 2015). 
Características relacionadas ao crescimento e desenvolvimento corporal de peixes de corte, como a tilápia do Nilo, são estudadas devido a sua importância econômica, vindo a contribuir com informações relevantes para a indústria do pescada e caracterização de uma espécie (HE et al., 2018).

No cenário da piscicultura ornamental, estudos abordando o tema são escassos, não havendo relatos publicados sobre a correlação entre características morfométricas e peso de peixes Betta splendens.

Peixes ornamentais podem ser submetidos a situações que favorecem o estresse como o transporte (NEVES et al., 2018), quando mantidos sobre altas densidades de estocagem (ABE et al., 2016) ou quando alimentados com dietas de baixa qualidade, fator que pode favorecer o aparecimento de doenças aumento da mortalidade (ZUANON et al., 2011).

Neste âmbito, Marengon et al. (2015), destacam que a glicose sanguínea pode ser utilizada como um indicador de estresse na produção de peixes, visto que, seu aumento expressivo pode indicar maior mobilização de glicose para suprir as necessidades do peixe em manter a homeostase.

Mediante o exposto, a pesquisa foi desenvolvida com o objetivo de descrever características fenotípicas e a concentração de glicose sanguínea $(\mathrm{dm} / \mathrm{L})$ de acordo com o sexo, como também, identificar correlações entre peso corporal e medidas morfométricas do Betta splendens.

\section{MATERIAL E MÉTODOS}

O experimento foi realizado no Laboratório de Aquicultura do Centro de Ciências Humanas, Sociais e Agrárias (CCHSA) da Universidade Federal da Paraíba, situado no município de Bananeiras, Paraíba - Brasil.

Foram utilizados 80 Bettas splendens divididos em dois grupos, machos e fêmeas, onde cada animal foi considerado como uma unidade experimental. Estes, apresentavam nadadeira crowntail, coloração azul escuro, 6 meses de vida e foram obtidos a partir da desova simultânea de 3 casais com características fenotípicas semelhantes.

Os machos foram separados do lote quando começaram a manifestar comportamentos agonísticos como ameaça (caracterizada pela abertura da boca, opérculos e nadadeiras frente a outro indivíduo) e luta (união das bocas para desafio de força).

Assim que foram identificados, os machos permaneceram alojados em recipientes 
plásticos transparentes individuais com capacidade para 2 litros. Nas laterais dos recipientes foram inseridas divisórias impedindo contato visual entre machos para evitar manifestação de comportamento agressivo. As fêmeas permaneceram alojadas em tanque de polietileno com capacidade para 100 litros.

Durante o período de adaptação de 15 dias, os peixes foram alimentados 3 vezes ao dia, até saciedade aparente, com ração comercial extrusada própria para a espécie. Semanalmente foi realizada a limpeza dos recipientes e tanque.

Os parâmetros físico-químicos da água: concentração de oxigênio dissolvido, pH e temperatura da água foram mensurados no dia da coleta de dados, com o auxílio de peagâmetro (HANNA®) e Oxímetro/termômetro (ICEL®). O teor de amônia dissolvido na água foi avaliado utilizando um kit colorimétrico comercial. Os valores obtidos foram: Oxigênio dissolvido $5,24 \mathrm{mg} / \mathrm{L} ; \mathrm{pH} 7,90$; temperatura $25,6{ }^{\circ} \mathrm{C}$ e concentração de amônia total 0,63 ppm.

Os peixes permaneceram em jejum por um período de 12 horas, sendo posteriormente anestesiados por banho de imersão em solução de benzocaína, pesados em balança analítica e medidos com paquímentro digital para obtenção do peso, comprimento total (medido da boca ao final da nadadeira caudal), comprimento padrão (medido da boca ao início da nadadeira caudal), altura (medida horizontalmente à frente da nadadeira dorsal e após nadadeira pélvica), largura (medida lateralmente após nadadeiras peitorais) e comprimento da cabeça (medido da boca ao final do opérculo), seguindo metodologia descrita por Gomes et al. (2018).

Para a coleta de sangue os peixes dos dois grupos, machos e fêmeas, foram mantidos em estágio de anestesia cirúrgica, sendo realizado corte com bisturi na região dorsal ao final da cabeça, para atingir a artéria aorta. A glicose foi mensurado em seguida pelo método da glicose desidrogenase flavina-adenina dinucleotídeo (GDH-FAD), por meio de glicosímetro portátil Bayer ContourTM TS e tiras reagentes.

Os dados foram submetidos à análise de variância, as médias comparadas pelo teste t ao nível de $5 \%$ de probabilidade e, posteriormente, análise de correlação de Pearson entre peso e características morfométricas foi realizada por meio do programa estatístico ASSISTAT 7.7.

\section{RESULTADOS E DISCUSSÃO}

O peso corporal dos machos e fêmeas Betta splendens avaliados não diferiram 
entre si, no entanto, o comprimento total foi maior em machos $(43,071 \mathrm{~mm})$ quando comparados às fêmeas $(36,242 \mathrm{~mm})$. O comprimento padrão, que exclui a medida da nadadeira caudal, não apresentou diferenças entre os sexos, evidenciando o maior crescimento das nadadeiras observado nos machos (Tabela 1).

Tabela 1 - Peso, medidas morfométricas e glicose sanguínea de machos e fêmeas Betta splendens

\begin{tabular}{llll}
\hline \multicolumn{1}{c}{ Variáveis } & Macho & Fêmeas & CV\% \\
\hline Peso $(\mathrm{g})$ & $0,8362^{\mathrm{a}}$ & $0,7994^{\mathrm{a}}$ & 29,44 \\
Comprimento Total $(\mathrm{mm})$ & $43,071^{\mathrm{a}}$ & $36,242^{\mathrm{b}}$ & 9,05 \\
Comprimento Padrão $(\mathrm{mm})$ & $29,771^{\mathrm{a}}$ & $28,463^{\mathrm{a}}$ & 8,59 \\
Altura $(\mathrm{mm})$ & $8,940^{\mathrm{a}}$ & $9,467^{\mathrm{a}}$ & 9,51 \\
Largura $(\mathrm{mm})$ & $5,354^{\mathrm{b}}$ & $6,394^{\mathrm{a}}$ & 10,76 \\
Comprimento da Cabeça $(\mathrm{mm})$ & $8,784^{\mathrm{a}}$ & $7,829^{\mathrm{b}}$ & 8,88 \\
Glicose sanguínea $(\mathrm{dm} / \mathrm{l})$ & $51,11^{\mathrm{a}}$ & $50,40^{\mathrm{a}}$ & 41,14 \\
\hline
\end{tabular}

*Médias na mesma linha seguidas de letras distintas diferem $(P \leq 0,05)$ pelo teste $t$.

A altura do corpo não diferiu entre os sexos, todavia Bettas machos têm menor largura $(5,354 \mathrm{~mm})$ e maior comprimento de cabeça $(8,784 \mathrm{~mm})$.

O aumento do comprimento da cabeça observado nos machos pode estar associado à necessidade de parecer maior que seu oponente durante encontros agonísticos, pois, como observado por Santos et al. (2018) quando submetidos a exposição de outro macho ou ao seu reflexo no espelho, os peixes Betta splendens tendem a abrir opérculos e nadadeiras em um comportamento ameaçador.

Não houve diferença significativa para a glicose sanguínea relacionada ao sexo, permanecendo entre 50,40 e 51,11 dm/l. Peixes carnívoros, mesmo quando alimentados com ração comercial, que contém ingredientes de origem vegetal, tendem a permanecer com baixas concentrações de glicose no sangue, quando comparados aos peixes onívoros. Hoshino et al. (2017) identificaram no peixe carnívoro pirarucu, com peso médio de $2 \mathrm{~kg}$

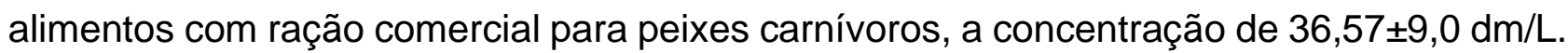
Em outra espécie de peixe ornamental, o cascudo (Peckoltia oligospila), níveis basais de 
glicose sanguínea permanecem entre 40 e 50 md/L (NEVES et al., 2018).

Peixes carnívoros geralmente possuem uma concentração de glicose mais baixa quando comparados a peixes onívoros. Hoshino et al. (2017) identificaram que a concentração de glicose sérica em pirarucus com peso médio de $2 \mathrm{~kg}$ alimentos com ração

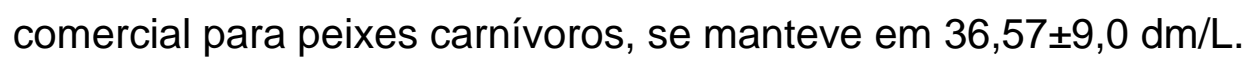

A análise de correlação de Pearson demonstrou que todas as variáveis analisadas apresentaram forte correlação $(P<0,01)$ quando comparadas, no entanto, o peso das fêmeas Betta splendens apresentou as mais altas correlações positivas com o comprimento total $(0,94)$, comprimento padrão $(0,91)$ e altura $(0,91)$. Foi possível observar também alta correlação positiva entre comprimento total e comprimento padrão $(0,97)$, sendo esta a correlação mais forte observada entre as variáveis analisadas, significando que à medida que o peso aumenta, maior será o comprimento total das fêmeas (tabela 2).

Tabela 2 - Correlação de Pearson entre as características peso, comprimento total, comprimento padrão, altura, largura e comprimento da cabeça de fêmeas Betta splendens

\begin{tabular}{|c|c|c|c|c|c|c|}
\hline Variáveis & Peso & CT & CP & ALT & LAR & CC \\
\hline Peso $(\mathrm{g})$ & 1 & $0,9422^{\star *}$ & $0,9178^{* *}$ & $0,9165^{\star \star}$ & $0,7801^{* *}$ & $0,8442^{* *}$ \\
\hline CT $(\mathrm{mm})$ & & 1 & $0,9746^{* *}$ & $0,8929^{* *}$ & $0,7421^{* *}$ & $0,8479^{* *}$ \\
\hline $\mathrm{CP}(\mathrm{mm})$ & & & 1 & $0,8964^{\star *}$ & $0,7497^{* *}$ & $0,8420^{* *}$ \\
\hline ALT (mm) & & & & 1 & $0,8196^{\star *}$ & $0,8410^{* *}$ \\
\hline LAR (mm) & & & & & 1 & $0,7765^{\star *}$ \\
\hline $\mathrm{CC}(\mathrm{mm})$ & & & & & & 1 \\
\hline
\end{tabular}

CT - Comprimento total; CP - Comprimento Padrão; ALT - Altura; LAR - Largura; CC Comprimento da cabeça. ${ }^{* *}$ significativo ao nível de $1 \%$ de probabilidade $(p<0,01)$.

Forte correlação positiva entre peso e comprimento total também foram identificadas em espécies de corte, como a traíra (0,97) (ARAÚJO et al., 2018) e o peixe carnívoro pirarucu $(0,93)$ (LUXINGER et al., 2018). Em outro estudo realizado com pintado foi possível verificar estreita correlação entre as variáveis peso total e comprimento total, com valores próximos de 1,0 sugerindo que o crescimento dos peixes tende a ser um crescimento isométrico (SCORVO FILHO et al., 2008). 
A relação entre ganho de peso e comprimento nos peixes pode ser avaliada através de variações de peso esperado em função do comprimento de um indivíduo ou de um grupo, indicando assim a sua condição corporal, visto que, uma ótima relação entre peso e comprimento pode indicar bem-estar positivo e bom desenvolvimento gonadal (MIRANDA e RIBEIRO, 1997; SCORVO FILHO et al., 2008).

As correlações de Pearson realizadas para avaliar a relação entre peso, comprimento padrão, comprimento total, altura, largura e comprimento da cabeça de peixes Betta splendens machos, evidenciaram que existe forte correlação positiva entre as variáveis, no entanto, as mais altas foram observadas entre peso e comprimento total $(0,92)$; peso e largura $(0,91)$; comprimento total e altura; largura e comprimento total $(0,93)$, sendo a mais forte entre comprimento da cabeça e comprimento total $(0,94)$, indicando que quanto maior for o peixe, maior será o comprimento de sua cabeça (tabela 3 ).

Tabela 3 - Correlação de Pearson entre as características peso, comprimento total, comprimento padrão, altura, largura e comprimento da cabeça de machos Betta splendens

\begin{tabular}{lcccccc}
\hline Variáveis & Peso & CT & CP & ALT & LAR & CC \\
\hline Peso $(\mathrm{g})$ & 1 & $0,9275^{\star *}$ & $0,8688^{\star *}$ & $0,8724^{\star *}$ & $0,9197^{\star *}$ & $0,8768^{\star *}$ \\
CT $(\mathrm{mm})$ & & 1 & $0,8985^{\star *}$ & $0,9245^{\star *}$ & $0,9307^{\star *}$ & $0,9413^{\star *}$ \\
CP $(\mathrm{mm})$ & & & 1 & $0,8554^{* *}$ & $0,7945^{\star *}$ & $0,8381^{\star *}$ \\
ALT $(\mathrm{mm})$ & & & & 1 & $0,8808^{\star *}$ & $0,8711^{\star *}$ \\
LAR $(\mathrm{mm})$ & & & & & 1 & $0,8703^{\star *}$ \\
CC $(\mathrm{mm})$ & & & & & & 1 \\
\hline
\end{tabular}

CT - Comprimento total; CP - Comprimento Padrão; ALT - Altura; LAR - Largura; CC Comprimento da cabeça. ${ }^{* *}$ significativo ao nível de $1 \%$ de probabilidade $(p<0,01)$.

A intensidade das correlações de Pearson para as variáveis observadas entre machos e fêmeas evidenciou que existe forte relação positiva entre todas as variáveis observadas, sendo a relação entre comprimento total e comprimento padrão $(0,97)$ a mais forte observada no grupo das fêmeas, enquanto no grupo dos machos a relação mais forte foi identificada entre comprimento total e comprimento de cabeça $(0,94)$.

Conhecer o comportamento e relação entre características fenotípicas do Betta 
splendens é de suma importância devido a sua posição no mercado pet, pois, pode contribuir para estudos visando seleção de características desejáveis nas espécies ornamentais, contribuindo com a formação de novas linhagens que atendam às exigências do consumidor final.

\section{CONCLUSÕES}

Os machos possuem maior comprimento total e de cabeça, enquanto as fêmeas apresentam maior largura. A concentração de glicose sanguínea varia entre 50,40 e 51,11 $\mathrm{dm} / \mathrm{l}$ para a espécie.

Existe forte correlação positiva entre todas as variáveis analisadas, sendo a relação entre comprimento total e comprimento padrão a mais forte observada no grupo das fêmeas, e a relação entre comprimento total e comprimento de cabeça para machos.

\section{REFERÊNCIAS}

ARAUJO, K. C.; CIRNE, L. G. A.; SOUZA, W. S. et al. Características morfométricas, rendimento de filé e composição química da traíra. Agroecossistemas, v. 10, n. 2, p. 2536, 2018.

CAVAlCANTI, L. P. D. G.; PONTES, R. J. S.; REGAZZI, A. C. F. et al. Competência de peixes como predadores de larvas de Aedes aegypti, em condições de laboratório. Revista de saúde pública, v. 41, n. 4, p. 638-644, 2007.

peixe Bettasplendens (Regan 1910). Revista Brasileira de Reprodução Animal, Belo Horizonte, v.30, n.3/4, p.134-149, 2006.

FOSSE, P.J.; MATTOS, D.C.; CARDOSO, L.D. et al. Estratégia de coalimentação na sobrevivência e no crescimento de larvas de Betta splendens durante a transição alimentar. Arquivo Brasileiro de Medicina Veterinária e Zootecnia, v.65, p.1801-1807, 2013.

HE, J.; ZHAO, Y.; ZHAO, J.; GAO, J.; XU, P.; YANG, R. Random regression analysis for body weights and main morphological traits in genetically improved farmed tilapia (Oreochromis niloticus). Journal of applied genetics, v. 59, n. 1, p. 99-107, 2018. 
HOSHINO, M. D. F. G.; MARINHO, R. D. G. B.; PEREIRA, D. F.; YOSHIOKA, E. T. O.; DIAS, M. T.; OZORIO, R. O. D. A.; FARIA, F. S. E. D. V. Hematological and biochemical responses of pirarucu (Arapaima gigas, Arapaimidae) fed with diets containing a glucomannan product derived from yeast and algae. Acta Amazônica, v. 47, n. 2, p. 87-94, 2017.

LUXINGer, A. O., CAVAlI, J., PORTO, M. O., SAleS-Neto, H. M., LAGO, A. A., FREITAS, R. T. F. Morphometric measurements applied in the evaluation of Arapaima gigas body components. Aquaculture, v.489, p 80-84. 2018.

MARENGONI, N. G., WEISS, L. A., ALBUQUERQUE, D. M., MOURA, M. C. Influência de probióticos na prevalência parasitária e níveis de glicose e cortisol em tilápia do Nilo. Archivos de zootecnia, v. 64, n. 245, p. 63-69, 2015.

MCDOWALL, R. M. Shoot first, and then ask questions: a look at aquarium fish imports and invasiveness in New Zealand. New Zealand Journal of Marine and Freshwater Research, v. 38, n. 3, p. 503-510, 2004.

MIRANDA, M.O.T. e RIBEIRO, P.L. Características zootécnicas do surubim. In: MIRANDA, M.O. T (Org). Surubim. Belo Horizonte: IBAMA, (Coleção Meio Ambiente. Série Estudos Pesca). 1997, p. $43-56$.

NEVES, M. S.; COUTO, M. V. S.; SOUSA, N. C.; SANTOS, R. F. B.; DIAS, H. M.; ABE, H. A.; DIAS, J.A.R.; CUNHA, F. S.; DIAS, M. T.; FUJIMOTO, R.Y. Resposta hematológica do cascudo ornamental amazônico Peckoltia oligospila ao estresse de transporte. Arquivo Brasileiro de Medicina Veterinária e Zootecnia, v.70, n. 1, p. 13 - 19, 2018.

OLIVEIRA, C. A. L.; YOSHIDA, G. M.; OLIVEIRA, S. N.; KUNITA, N. M.; SANTOS, A. I.; ALEXANDRE FILHO, L.; RIBEIRO, R. P. Avaliação genética de tilápias-do-nilo durante cinco anos de seleção. Pesquisa Agropecuária Brasileira, v. 50, n. 10, p. 871-877, 2015.

SANTOS, E. L.,; SILVA, L. L. A.; SOARES, E. C.; DUARTE, A. G.; SILVA, T. J.; MEDEIROS, E. S.; SANTOS FERREIRA, A. J. Efeito da cor do ambiente sobre o comportamento social de machos de Betta splendens. Nucleus, v. 15, n. 1, p. 51-60, 2018. 
SCORVO FILHO, J.D.; ROMAGOSA, E.; AYROZA, L.M.S.; FRASCÁ-SCORVO, C.M.D. Desempenho produtivo do pintado, Pseudoplatystoma corruscans (spix \& agassiz, 1829), submetidos a diferentes densidades de estocagem em dois sistemas de criação: intensivo e semi-intensivo. Boletim do Instituto de Pesca, v.34, n.2, p.181 - 188, 2008.

WHITTINGTON, R.J.; CHONG, R. Global trade in ornamental fish from an Australian perspective: the case for revised import risk analysis and management strategies. Preventive Veterinary Medicine, v.81, n. 1 -3, p. 92-116, 2007. 